\title{
Recent results from SND detector at VEPP-2000 collider.
}

M. N. Achasov ${ }^{1,2, *}$, A. Yu. Barnyakov ${ }^{1,2}$, K. I. Beloborodov ${ }^{1,2}$, A. V. Berdyugin ${ }^{1,2}$,

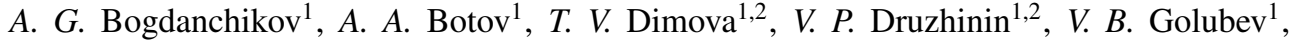
L. V. Kardopoltsev ${ }^{1,2}, A$. G. Kharlamov ${ }^{1,2}, A$. N. Kirpotin ${ }^{1}, A . A$. Korol ${ }^{1,2}, S$. V. Koshuba ${ }^{1}$, D. P. Kovrizhin ${ }^{1}$, A. S. Kupich ${ }^{1,2}$, R. A. Litvinov ${ }^{1}$, A. P. Lysenko ${ }^{1}$, K. A. Martin ${ }^{1}$, N. A. Melnikova ${ }^{1,2}$, N. Yu. Muchnoi ${ }^{1,2}$, A. E. Obrazovsky ${ }^{1}$, E. V. Pakhtusova ${ }^{1}$, E. A. Perevedentsev ${ }^{1,2}, K . \quad$ V. Pugachev ${ }^{1,2}$, S. I. Serednyakov ${ }^{1,2}$, D. N. Shatilov ${ }^{1}$, P. Yu. Shatunov ${ }^{1}, Y u$. M. Shatunov ${ }^{1}$, D. A. Shtol ${ }^{1,2}, D$. B. Shwartz ${ }^{1,2}, Z$. K. Silagadze ${ }^{1,2}$, A. N. Skrinsky ${ }^{1}, I$. K. Surin ${ }^{1}, Y u$. V. Usov ${ }^{1}, I$. M. Zemlyansky ${ }^{1,2}$, and V. N. Zhabin ${ }^{1,2}$

${ }^{1}$ Budker Institute of Nuclear Physics, SB RAS, Novosibirsk, 630090, Russia

${ }^{2}$ Novosibirsk State University, Novosibirsk, 630090, Russia

\begin{abstract}
Recent results on $e^{+} e^{-}$annihilation to hadrons from SND experiment at VEPP-2000 collider are presented. Corresponding data set is about $200 \mathrm{pb}^{-1}$ of integrated luminosity collected at the energy region $\sqrt{s}$ below $2 \mathrm{GeV}$. The processes $e^{+} e^{-} \rightarrow \pi^{+} \pi^{-}, \pi^{0} \gamma, \omega \pi^{0}, \pi^{+} \pi^{-} \pi^{0}, \eta \pi^{+} \pi^{-} \pi^{0}, n \bar{n}, \pi^{+} \pi^{-} 4 \pi^{0}$ have been studied. Searches for $e^{+} e^{-}$annihilation to $C$-even resonances $\eta$ and $f_{1}(1285)$ were done.
\end{abstract}

\section{Introduction}

The spherical neutral detector SND [1,2] is a general purpose nonmagnetic detector. SND consists of the tracking system based on cylindrical drift and proportional chambers placed in a common gas volume, aerogel threshold counters [3], calorimeter and muon system based on proportional tubes and plastic scintillator. The main part of SND is a three-layer spherical electromagnetic calorimeter based on $\mathrm{NaI}(\mathrm{Tl})$ crystals. The total calorimeter thickness for particles originating from the interaction region is $13.4 X_{0}$ of $\mathrm{NaI}(\mathrm{Tl})$. The total number of counters is 1632 .

SND is operating at VEPP-2000 [4] $e^{+} e^{-}$collider and collected about $200 \mathrm{pb}^{-1}$ in the center-of-mass energy range from 0.2 to $2.0 \mathrm{GeV}$. The beam energy is measured using the Compton scattering of the laser photons on the electron beam [5].

Experimental studies include measurement of the cross sections of the $e^{+} e^{-}$annihilation to hadrons, study of the light vector mesons $\rho, \omega, \phi$ and their excited states, search for processes of $e^{+} e^{-}$annihilation to $C$-even resonances. Here we report results of $e^{+} e^{-} \rightarrow \pi^{+} \pi^{-}$, $\pi^{0} \gamma, \omega \pi^{0}, \pi^{+} \pi^{-} \pi^{0}, \eta \pi^{+} \pi^{-} \pi^{0}, n \bar{n}, \pi^{+} \pi^{-} 4 \pi^{0}, \eta$ and $f_{1}(1285)$ processes studies.

\section{$2 e^{+} e^{-}$annihilation into two particles.}

The $e^{+} e^{-} \rightarrow \pi^{+} \pi^{-}$cross section was measured in the energy range $\sqrt{s}=0.5-1 \mathrm{GeV}$. The measurement is based on data with an integrated luminosity of $5 \mathrm{pb}^{-1}$.

\footnotetext{
*e-mail: achasov@inp.nsk.su
} 
The analysis was based on selection of the collinear events $e^{+} e^{-} \rightarrow e^{+} e^{-}, \pi^{+} \pi^{-}, \mu^{+} \mu^{-}$. The cosmic background was rejected by veto of the muon system. Using energy depositions in the calorimeter's counters the selected events were divided into two classes $e^{+} e^{-} \rightarrow e^{+} e^{-}$ and $e^{+} e^{-} \rightarrow \pi^{+} \pi^{-}, \mu^{+} \mu^{-}$. The events separation was based on the machine learning approach [6]. The $e^{+} e^{-} \rightarrow e^{+} e^{-}$events were used to obtain integrated luminosity. The number of $e^{+} e^{-} \rightarrow \mu^{+} \mu^{-}$events was subtracted according to QED calculation. The measured preliminary cross section (fig.1) was fitted by VDM taken into account $\rho, \omega$ and $\rho^{\prime}$ resonances. The systematical error of the cross section determination is about $0.8-0.9 \%$.

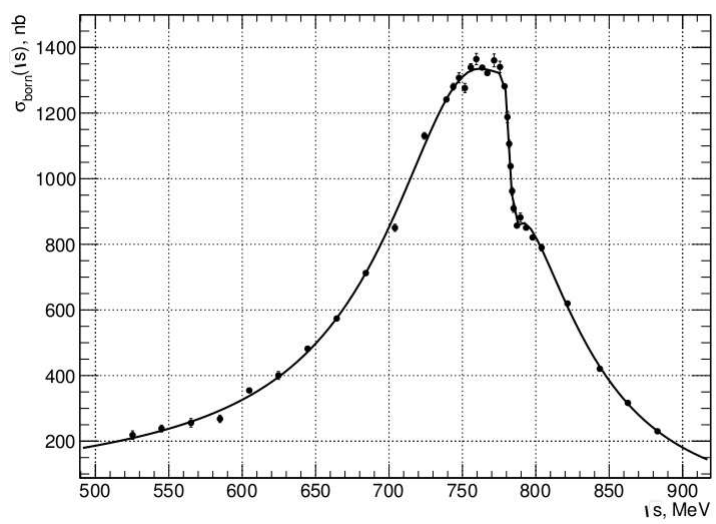

Figure 1. The preliminary cross section of the process $e^{+} e^{-} \rightarrow \pi^{+} \pi^{-}$measured by SND. The curve is the VDM fit.

Previously the $e^{+} e^{-} \rightarrow \pi^{0} \gamma$ cross section was measured below $1.4 \mathrm{GeV}$ by SND [8-10] and CMD-2 [11] at VEPP-2M. Here we present a result based on the integrated luminosity of about $40 \mathrm{pb}^{-1}$ collected by SND at VEPP-2000 at the energy range $\sqrt{s}=1.075-2 \mathrm{GeV}$ [12]. In the energy range $\sqrt{s}=1.4-2 \mathrm{GeV}$ the process $e^{+} e^{-} \rightarrow \pi^{0} \gamma$ was studied for the first time.

In analysis three-photon events of the process $e^{+} e^{-} \rightarrow \pi^{0} \gamma \rightarrow 3 \gamma$ and two-photon events of the process $e^{+} e^{-} \rightarrow \gamma \gamma$ which was used for normalization were selected simultaneously. Some selection criteria, such as absence of charged tracks in an event and the muon-system veto, are common for both processes, and systematic uncertainties associated with these criteria cancel as a result of the normalization. Final selection of $e^{+} e^{-} \rightarrow 3 \gamma$ events was based on $4 \mathrm{C}$ kinematic fit. To determine the number of signal events, the spectrum of the mass recoiling against the most energetic photon in an event was fitted by a sum of signal and background distributions (fig.2).

The measured cross section is shown in fig.3. Below $1.4 \mathrm{GeV}$ the cross section is about 50 $\mathrm{pb}$ and agrees with previous SND and CMD-2 measurements at VEPP-2M [10, 11]. Above $1.4 \mathrm{GeV}$ it is consistent with zero within statistical errors of about $15 \mathrm{pb}$. To explain the $e^{+} e^{-} \rightarrow \pi^{0} \gamma$ cross section beside $\rho, \omega$ and $\phi$ resonances (dashed line in fig. 3 ) the contributions of $\rho^{\prime}$ and $\omega^{\prime}$ resonances are required with significance of $4 \sigma$. This significance was obtained using all available data [10-12].

Previously the $e^{+} e^{-} \rightarrow n \bar{n}$ cross section was measured below $2 \mathrm{GeV}$ by SND [13] at VEPP-2000 collider with the integrated luminosity about $10 \mathrm{pb}^{-1}$ collected in 2011-2012 and by FENICE [14] experiments. A new result based on the integrated luminosity about $20 \mathrm{pb}^{-1}$ 


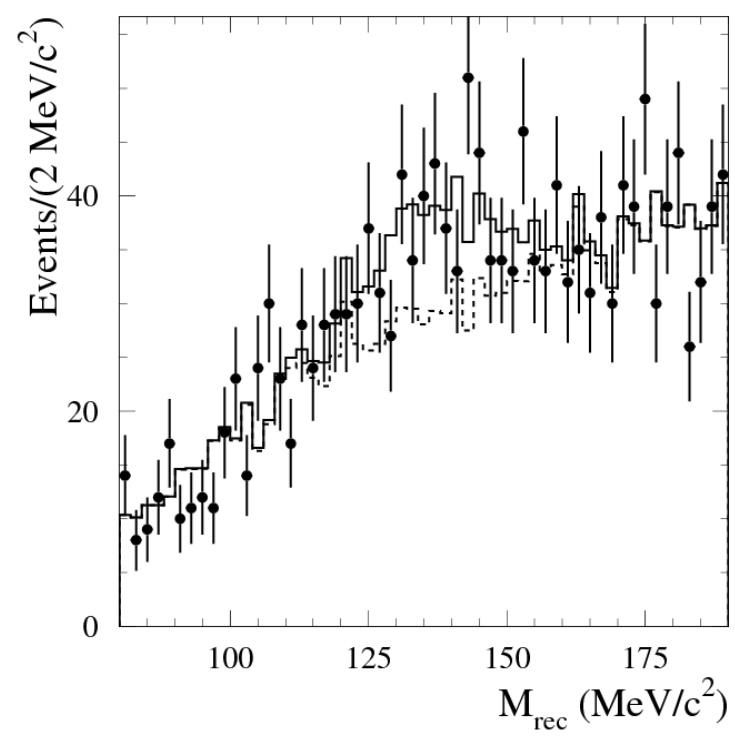

Figure 2. The recoil mass $M_{r e c}$ distribution at $\sqrt{s}=1075-1375 \mathrm{MeV}$ for data events (points with error bars) together with result of the fit (solid histogram). The dashed histogram shows the background distribution.

collected by SND at VEPP-2000 in 2017 can be found in contribution by S.I.Serednyakov «Measurement of the $e+e \rightarrow n \bar{n}$ cross section with the SND detector at the VEPP-2000 collider» in these proceedings.

\section{Multihadron processes.}

The process $e^{+} e^{-} \rightarrow \omega \pi^{0}$ is one of the dominant in the energy region above $\phi$-meson. It was studied using $\pi^{+} \pi^{-} 2 \pi^{0}$ final state with the integrated luminosity about $40 \mathrm{pb}^{-1}$. In analysis events with two charged tracks and at least four photons were used. Final selection of $e^{+} e^{-} \rightarrow$ $\pi^{+} \pi^{-} 2 \pi^{0}$ events was based on kinematic fit, which takes into account the energy-momentum conservation and constraints on the invariant masses of the photons pairs to be equal to the $\pi^{0}$ mass. The number of signal events was determined from the fit of the $\pi^{+} \pi^{-} \pi^{0}$ invariant mass spectrum. The preliminary result of the cross section measurement is shown in fig.4.

The process $e^{+} e^{-} \rightarrow \pi^{+} \pi^{-} \pi^{0}$ was studied in the energy range from 1050 to $2000 \mathrm{MeV}$. The measured cross section is shown in fig.5. The red points are already published result [20] and the blue points are the new one. The contributions of $\omega^{\prime}$ and $\omega^{\prime \prime}$ are clearly seen.

The dynamics of the $e^{+} e^{-} \rightarrow \pi^{+} \pi^{-} \pi^{0} \eta$ final state includes the following mechanisms: $\omega \eta, \phi \eta, a_{0}(980) \rho$ and structureless $\pi^{+} \pi^{-} \pi^{0} \eta$ [21-24]. The analysis was based on events with $\eta \rightarrow \gamma \gamma$ decay. The number of $e^{+} e^{-} \rightarrow \pi^{+} \pi^{-} \pi^{0} \eta$ events is determined from the fit to the $\eta$-meson candidates $\gamma \gamma$ invariant mass spectrum. The cross sections of the processes $e^{+} e^{-} \rightarrow$ $\omega \eta, \phi \eta$ and $a_{0}(980) \rho$ plus structureless $\pi^{+} \pi^{-} \pi^{0} \eta$ were obtained. More details are available in contribution «Measurement of the $e^{+} e^{-} \rightarrow \pi^{+} \pi^{-} \pi^{0} \eta$ cross section below $\sqrt{s}=2 \mathrm{GeV} »$ by A.A. Botov to these proceedings.

The process $e^{+} e^{-} \rightarrow \pi^{+} \pi^{-} 4 \pi^{0}$ below $2 \mathrm{GeV}$ was studied for the first time. The preliminary result of the cross section measurement is shown in fig. 6 . 


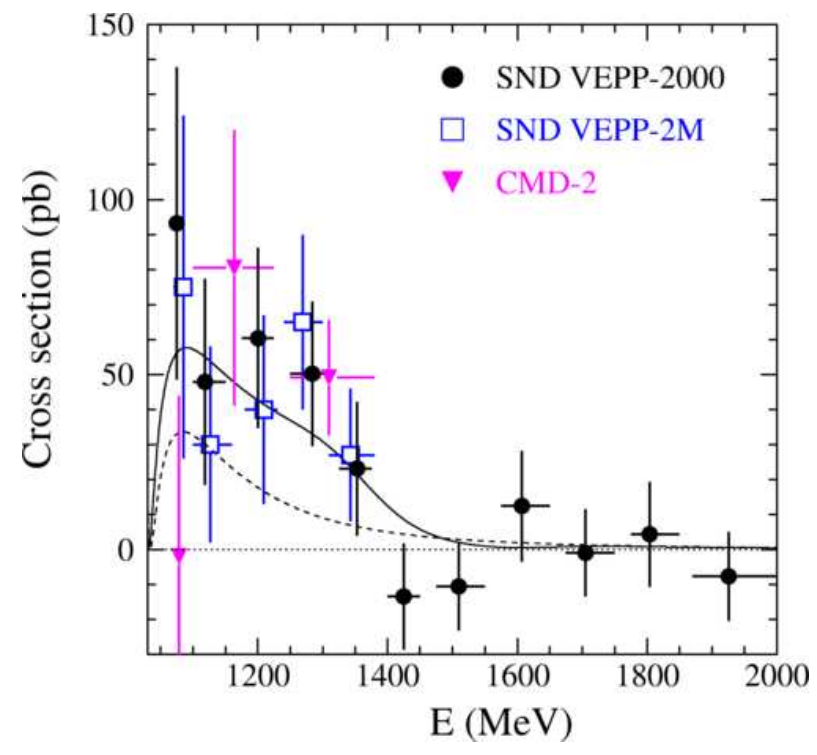

Figure 3. The $e^{+} e^{-} \rightarrow \pi^{0} \gamma$ cross section measured by SND [12] in the energy range $\sqrt{s}=1.075-$ $2 \mathrm{GeV}$ together with the previous most accurate measurements CMD-2 [11] and SND VEPP-2M [10]. The solid curve is VDM fit taking into account $\rho, \omega, \phi, \rho^{\prime}$ and $\omega^{\prime}$. The dashed line shows contribution of $\rho, \omega$ and $\phi$ only. Only statistical errors are shown.

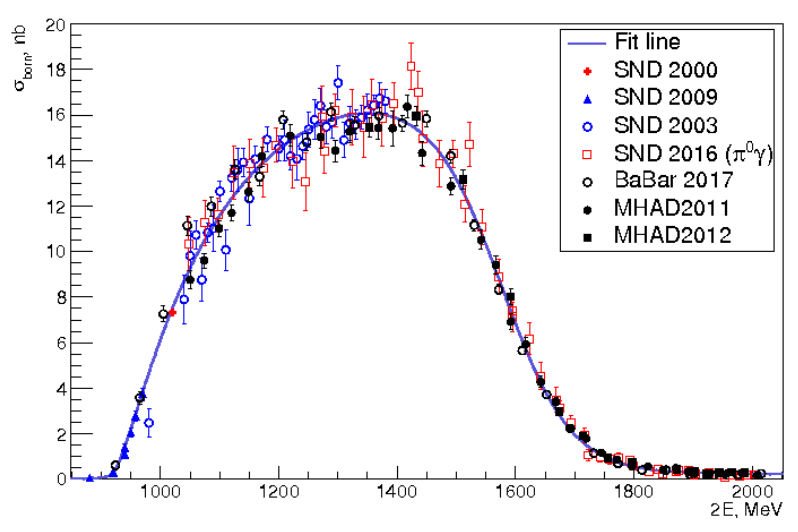

Figure 4. The $e^{+} e^{-} \rightarrow \omega \pi^{0}$ preliminary cross section measured by SND (black points and squares) in the energy range $\sqrt{s}=1.05-2 \mathrm{GeV}$ together with the previous measurements of SND and BaBar [15-19]. The solid curve is VDM fit.

\section{Search for $e^{+} e^{-}$annihilation to $C$-even resonances.}

A search for the rare decay $\eta \rightarrow e^{+} e^{-}$was performed using the inverse process $e^{+} e^{-} \rightarrow \eta$. The integrated luminosity about $650 \mathrm{nb}^{-1}$ collected by SND in 2018 at the energy $\sqrt{s}=$ $m_{\eta}=547.865 \pm 0.017 \mathrm{MeV}$ was used. The beam energy was measured by the Compton backscattering method. The events $e^{+} e^{-} \rightarrow \eta \rightarrow 3 \pi^{0} \rightarrow 6 \gamma$ were selected. The detection efficiency was equal to $14 \%$ and zero events was found. The upper limit $\operatorname{Br}\left(\eta \rightarrow e^{+} e^{-}\right)<$ 


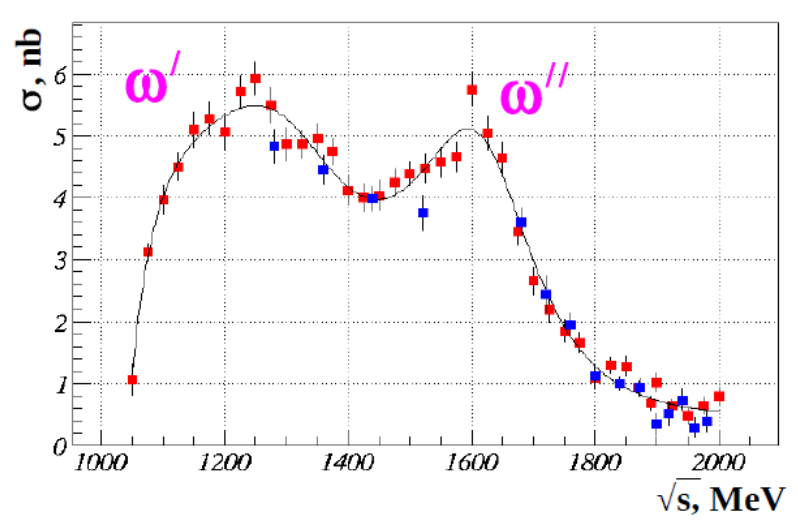

Figure 5. The $e^{+} e^{-} \rightarrow \pi^{+} \pi^{-} \pi^{0}$ preliminary cross section measured by SND in the energy range $\sqrt{s}=$ $1.05-2 \mathrm{GeV}$. Red points [20], blue points is a new preliminary result. The solid curve is VDM fit.

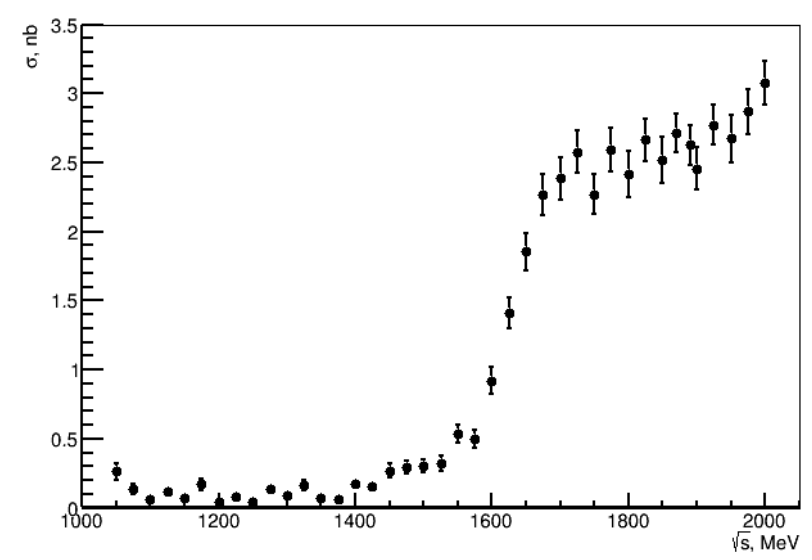

Figure 6. The $e^{+} e^{-} \rightarrow \pi^{+} \pi^{-} 4 \pi^{0}$ preliminary cross section measured by SND in the energy range below $2 \mathrm{GeV}$.

$7 \times 10^{-7} 90 \% \mathrm{CL}$ was set [25]. It is three times better then the previous limit $2.3 \times 10^{-6}$ [26], but still much higher then unitary limit prediction: $\operatorname{Br}\left(\eta \rightarrow e^{+} e^{-}\right)=1.78 \times 10^{-9}$ [27].

The process $e^{+} e^{-} \rightarrow f_{1}(1285)$ was studied for the first time. Theoretical VDM based calculation predicts: $\operatorname{Br}\left(f_{1}(1285) \rightarrow e^{+} e^{-}\right)=(3-8) \times 10^{-9}$ [28]. For experimental studies the integrated luminosity $3.4 \mathrm{pb}^{-1}$ accumulated at $\sqrt{s}=1285 \mathrm{MeV}$ was used. The analysis was based on the selection of $e^{+} e^{-} \rightarrow \eta \pi^{0} \pi^{0} \rightarrow 6 \gamma$ events. The main background is $e^{+} e^{-} \rightarrow$ $\pi^{0} \pi^{0} \gamma \rightarrow 5 \gamma$. Other background processes are $e^{+} e^{-} \rightarrow \omega \pi^{0} \pi^{0}, \omega \eta \pi^{0}, \eta \gamma \rightarrow 7 \gamma$. For signal events selection and the background suppression the kinematic fits with various hypothesis were used. The detection efficiency of $e^{+} e^{-} \rightarrow \eta \pi^{0} \pi^{0} \rightarrow 6 \gamma$ process was equal to $15 \%$. Two events was found, while the calculated background (mainly $e^{+} e^{-} \rightarrow \pi^{0} \pi^{0} \gamma$ ) is 1.2 event. The upper limit $\operatorname{Br}\left(f_{1}(1285) \rightarrow e^{+} e^{-}\right)<3.5 \times 10^{-9} 90 \%$ CL. was set. 


\section{Conclusions.}

During 2010-2018 the SND detector accumulated about $200 \mathrm{pb}^{-1}$ of the integrated luminosity at the VEPP-2000 electron-positron collider in the energy range $\sqrt{s}=0.2-2.0 \mathrm{GeV}$. The data taking is continued with a goal of $1 \mathrm{fb}^{-1}$ of the integrated luminosity. The data analysis on the hadron production is in progress. The obtained results have comparable or better accuracy than previous measurements. For several processes the cross sections have been measured for the first time.

This work is supported in part by the RFBR grants 18-02-00382-a, 18-02-00147-a.

\section{References}

[1] M.N.Achasov et. al., Nucl. Instr. Meth. A 449, 125 (2000)

[2] M.N. Achasov, et. al., Nucl. Phys. Proc. Suppl. 225-227, 66 (2012)

[3] A.Yu. Barnyakov, et. al., Prib. Tekh. Eksp. 1, 37 (2015)

[4] D.E. Berkaev et. al., Zh. Eksp. Teor. Fiz. 140, 247 (2011)

[5] E.V. Abacumova, et.al., Nucl. Instr. Meth. A744, 35 (2014)

[6] M.N. Achasov, K.I. Beloborodov, A.S. Kupich JINST 12, T01002 (2017)

[7] I. Logashenko, et. al., J. Phys. Chem. Ref. Data 44 (2015) 031211

[8] M.N.Achasov, et. al., Eur. Phys. J. C 12, 25 (2000)

[9] M.N.Achasov, et. al., Phys. Lett. B 559, 171 (2003)

[10] M.N.Achasov, et. al., Phys. Rev. D 93, 092001 (2016)

[11] R.R. Akhmetshin, et. al., Phys. Lett. B 605, 26 (2005)

[12] M.N.Achasov, et. al., Phys. Rev. D 98, 112001 (2018)

[13] M.N. Achasov, et. al., Phys. Rev. D 90, 112007 (2014)

[14] A. Antonelli, et. al., Nucl. Phys. B517, 3 (1998)

[15] V.M. Aulchenko, et. al., Zh. Eksp. Teor. Fiz. 117, 1067 (2000)

[16] M.N. Achasov, et. al., Zh. Eksp. Teor. Fiz. 123, 899 (2003)

[17] M.N. Achasov, et. al., Zh. Eksp. Teor. Fiz. 136, 442 (2009)

[18] M.N. Achasov, et. al., Phys. Rev. D 94, 112001 (2016)

[19] J.P. Lees, et. al., Phys. Rev. D 96, 092009 (2017)

[20] V.M. Aulchenko, et. al., Zh. Eksp. Teor. Fiz. 148, 34 (2015)

[21] B. Aubert, et al., Phys. Rev. D 73, 052003 (2006).

[22] M. N. Achasov, et al., Phys. Rev. D 94, 092002 (2016).

[23] V. P. Druzhinin, et al., EPJ Web Conf. 130, 05004 (2016)

[24] R. R. Akhmetshin, et al., Phys. Lett. B 773, 150 (2017)

[25] M.N. Achasov, et. al., Phys. Rev. D 98, 052007 (2018)

[26] G. Agakishiev, et al., Phys. Lett. B 731, 265 (2014)

[27] S. Berman and D. Geffen, Nuovo Cim. 18, 1192 (1960)

[28] A. S. Rudenko, Phys. Rev. D 96, 076004 (2017) 